\title{
Extended exposure to environmental cues, but not to sucrose, reduces sucrose cue reactivity in rats
}

\author{
John H. Harkness ${ }^{1}$ - Jason Wells ${ }^{1}$ - Sierra Webb ${ }^{1}$ - Jeffrey W. Grimm ${ }^{1,2}$
}

Published online: 14 July 2015

(C) Psychonomic Society, Inc. 2015

\begin{abstract}
In the present study, we examined the effects of extinction of sucrose-predictive contextual cues and/or sucrose satiation on the expression of sucrose cue reactivity in a rat model of relapse. Context extinction was imposed by housing rats in their home cage or in the operant conditioning chamber for $17 \mathrm{~h}$ prior to testing. For sucrose satiation, rats were allowed unlimited access to water or sucrose for $17 \mathrm{~h}$ prior to testing. Cue reactivity was assessed after either one (Day 1) or 30 (Day 30) days of forced abstinence from sucrose self-administration. An abstinence-dependent increase in sucrose cue reactivity was observed in all conditions ("incubation of craving"). Context extinction dramatically reduced lever responding on both Day 1 and Day 30. Sucrose satiation had no significant effect on cue reactivity in any condition. These results demonstrate that the context in which self-administration occurs maintains a powerful influence over cue reactivity, even after extended forced abstinence. In contrast, the primary reinforcer has little control over cue reactivity. These findings highlight the important role of conditioned contextual cues in driving relapse behavior.
\end{abstract}

Keywords Context $\cdot$ Cue reactivity $\cdot$ Extinction $\cdot$ Relapse . Satiety

Relapse is a major limitation in treating addictions. Craving induced by contact with cues previously associated with food

Jeffrey W. Grimm

jeff.grimm@wwu.edu

Western Washington University, Bellingham, WA, USA

2 Department of Psychology and Program in Behavioral Neuroscience, Western Washington University, 516 High Street, Bellingham, WA 98225-9172, USA or drugs ("cue reactivity") is an important factor in promoting relapse. In rodents, cue reactivity has been demonstrated extensively using operant and Pavlovian conditioning procedures (Rescorla, 2003; Robbins, Ersche, \& Everitt, 2008), and these behaviors have been used to model human relapse (Preston et al., 2009). The role of context in relapse has been explored previously. For example, in the "ABA" renewal procedure (Bouton \& Bolles, 1979; Crombag, Grimm, \& Shaham, 2002; Todd, Winterbauer, \& Bouton, 2012a, 2012b), responding is first reinforced in the " $A$ " context, then extinguished in the " $\mathrm{B}$ " context. Context-dependent cue reactivity is subsequently demonstrated as a reinstatement of responding in the " $A$ " context. " $A$ " comes to signal reinforcer availability, and "B," nonavailability (Crombag \& Shaham, 2002). This context-dependent cue reactivity reveals powerful occasion-setting properties of the operant conditioning chamber context. Targeting the occasion-setting properties of a food- or drug-predictive context may be a useful clinically. For example, exposure to a reward-paired context in the absence of the reward may benefit those recovering from drug dependence by reducing the power of the context to trigger relapse (Bedi et al., 2011). Exposure therapy has received attention in both the food (Dagher, 2009) and smoking (Freeman, Morgan, Beesley, \& Curran, 2012) literatures as a possible relapse prevention approach.

In the present study, we examined the importance of the self-administration context in a rat model of relapse (Shalev, Grimm, \& Shaham, 2002) by exposing subjects to the context for an extended period (overnight) following ten daily selfadministration training sessions. Relapse, or cue reactivity, was operationally defined as rats responding to a discrete tone+ light stimulus paired with sucrose delivery during training. Utilization of sucrose as a reinforcer models the reward circuitry activations and addiction behaviors common to drugs of abuse and food (Volkow \& Wise, 2005). Furthermore, this 
relapse model allows examination of "incubation of craving," an abstinence-dependent increase in cue reactivity that has recently been demonstrated to have translational significance (Bedi et al., 2011; Li et al., 2014; Wang et al., 2014), since cue reactivity may be assessed any number of days into abstinence from self-administration.

In summary, after ten days of sucrose self-administration, some rats underwent extinction in the self-administration context overnight. Some rats were also satiated to sucrose as a means to compare the relative effectiveness of prolonged exposure to the primary reinforcer, as opposed to the context of reinforcement, on reducing subsequent cue reactivity. These manipulations occurred immediately prior to cue reactivity testing one or 30 days into forced abstinence from sucrose self-administration.

\section{Method}

\section{Subjects}

The subjects were 66 male Long-Evans rats (Simonsen-derived, Gilroy, California, USA), weighed $419.8 \pm 7.1 \mathrm{~g}$ (mean $\pm S E M$ ), and were at least 3 months old at the start of the study. The rats were bred in the Western Washington University Psychology Department vivarium. Rats were weighed each Monday, Wednesday, and Friday for the duration of the experiment. The rats were given ad libitum access to Purina Mills Mazuri Rodent Pellets (Gray Summit, MO, USA), and water was provided ad libitum except where noted in General Procedures. Food and water were also available ad libitum in the operant conditioning chambers, except where noted in the General procedures section. All subjects lived singly housed in the vivarium except during the daily training or testing sessions, when they were brought to the operant conditioning chambers. The rats were maintained on a reverse 12:12-h light:dark cycle with lights off at 7 AM. All procedures followed the guidelines outlined in the "PHS Policy on Humane Care and Use of Laboratory Animals" (PHS, 2002) and were approved by the Western Washington University Institutional Animal Care and Use Committee.

\section{Apparatus}

Med Associates (St. Albans, VT, USA) operant conditioning chambers $(30 \times 20 \times 24 \mathrm{~cm})$ were controlled by Med PC software (Med Associates) and were enclosed in soundattenuating chambers with ventilation fans (Western Washington University). The operant conditioning chambers were outfitted with two levers $11 \mathrm{~cm}$ above the floor, a red house light, a 7.5-W white stimulus light, an infusion pump, and a liquid drop receptacle for delivery of sucrose. Each chamber was also equipped with four infrared photobeams
(Med Associates) that crisscrossed the chamber. Locomotor activity was recorded as the number of beam breaks per session.

\section{General procedures}

A training or testing session began with extension of the active lever and illumination of the house light. Presses on the active lever delivered $0.4 \mathrm{ml}$ of a $10 \%$ sucrose solution into the liquid drop receptacle on a "fixed-ratio 1" (FR1) schedule. This response also activated a 5-s compound stimulus consisting of a tone $(2 \mathrm{kHz}, 15 \mathrm{~dB}$ over ambient noise $)$ and illumination of the white stimulus light. A response on the inactive (nonretractable) lever was recorded, but did not have a programmed consequence. The experiment consisted of four phases: training, forced abstinence, exposure, and testing (Fig. 1).

Training phase Rats were water deprived in their home cages $17 \mathrm{~h}$ before the first training session and until they reached $>20$ sucrose deliveries/day or after the second day of selfadministration training. Water was not initially available in the operant conditioning chamber, but was added once water was returned to the home cage. Training was conducted in ten consecutive daily 2 -h sessions under a continuous reinforcement schedule (FR1) on the active lever. Rats were placed in the same particular operant conditioning chamber for all training, exposure, and testing conditions. Each presentation of the compound stimulus was followed by a 40 -s timeout, during which presses on the active lever were recorded but had no programmed consequence. The time out did not add excess time to the 2-h session. At the end of each session, the house light was turned off and the active lever was retracted. There was no limit to the number of reinforcers earned, other than that imposed by the length of the session (180 reinforcers maximum).

Forced-abstinence phase At the end of the training phase, subjects were randomly assigned to one of the forcedabstinence periods (one or 29 days), with the subsequent test session being referred to hereafter as Day 1 or Day 30 of forced abstinence. The rats lived in the vivarium for the duration of forced abstinence.

Exposure phase The rats in each forced-abstinence period condition were divided into four treatment groups: home cage + water, home cage + sucrose, operant conditioning chamber+water, and operant conditioning chamber+sucrose. At $17 \mathrm{~h}$ prior to testing, subjects were left in the home cage or placed in the operant conditioning chamber, according to their assignment, and provided with a bottle of either $200 \mathrm{ml} \mathrm{su}$ crose (10\% solution) or $200 \mathrm{ml}$ water. The subjects in the operant conditioning chamber condition were placed there 


\begin{tabular}{|c|c|c|}
\cline { 2 - 3 } \multicolumn{1}{c|}{} & \multicolumn{2}{l|}{ Forced Abstinence } \\
\hline Training & Exposure & Testing on Day 1 \\
\hline 10 days SA & $17 \mathrm{~h}$ & $\mathbf{2 h}$ \\
& & \\
\hline
\end{tabular}

\begin{tabular}{|c|c|c|c|}
\hline & Forced Abstinence & & \\
\hline Training & Home Cage & Exposure & Testing on Day 30 \\
\hline 10 days $S A$ & $\begin{array}{l}\text { Until 4PM } 29^{\text {th }} \text { Day of Forced } \\
\text { Abstinence }\end{array}$ & $17 \mathrm{~h}$ & $2 \mathrm{~h}$ \\
\hline
\end{tabular}

\section{Training}

$2 \mathrm{~h}$ daily sucrose self-administration (SA)

\section{Exposure}

Housed in either Home Cage or Operant Conditioning Chamber (extinction) with bottle of either water or sucrose (satiety)

\section{$\underline{\text { SA and Testing }}$}

Occurs in Operant Conditioning Chamber

Fig. 1 General procedure

without the presence of the house light, active lever, or compound stimulus. The doors of the sound-attenuating chambers were left slightly ajar to allow ambient light to penetrate (lights on at 7 PM, off at 7 AM). Ventilation fans were on during the chamber exposure period. Food was available in all conditions. Plain water was not provided during the $17-\mathrm{h}$ sucrose satiation period. At the end of the satiation period, both the water and sucrose bottles were removed and their contents measured and recorded.

Testing phase Testing was identical to training, except sucrose was not delivered for active-lever presses. At the conclusion of the testing phase, subjects were returned to the home cage.

\section{Analyses}

Training phase Active-lever presses, inactive-lever presses, sucrose deliveries, and photobeam breaks were analyzed separately using repeated measures (RM) analyses of variance (ANOVAs), with Days 7-10 of training as a within-group variable. Forced-Abstinence Day and Exposure Condition (housing and fluid assignment) were between-group factors. These between-group factors were included at this point to verify that the groups were similar in response rates prior to being assigned to experimental conditions.

Exposure phase In all, $17 \mathrm{~h}$ of sucrose and water consumption was analyzed using ANOVA with the between-group factors of Forced-Abstinence Day, Housing, and Fluid Assignment. All dependent measures were analyzed separately.

Testing phase Response data (active-lever presses, inactivelever presses, and compound-cue deliveries) and photobeam breaks were analyzed using an ANOVA with the same between-group factors identified above.

Statistics Sources of significant two-way interactions were examined by main effect analysis. $F$ values are only reported for significant interactions and main effects, unless nonsignificant main effects or interactions are necessary to illustrate a relevant lack of significance. The effect sizes of significant ANOVAs are reported as partial eta squared $\left(\eta_{\mathrm{p}}{ }^{2}\right)$. The Neuman-Keuls test was used for post-hoc comparison of means where appropriate. The alpha level was set at .05. All statistical analyses were conducted using the Statistica 12 software package (StatSoft, Inc., Tulsa, OK, USA). Figures were made using the SigmaPlot 8.0 software package (SPSS, Chicago, Il, USA).

\section{Results}

\section{Training phase}

We found significant main effects of training day (Days 7-10) on active-lever pressing $\left[F(3,195)=10.2, p<.0001, \eta_{\mathrm{p}}{ }^{2}=.14\right]$ and sucrose deliveries $\left[F(3,195)=9.0, p<.0001, \eta_{\mathrm{p}}{ }^{2}=.12\right]$, with active lever responding increasing over these days. Inactive lever responding did not change over these days $\left[F(3,195)=2.0, p=.1, \eta_{\mathrm{p}}{ }^{2}=.03\right]$. There were no significant interactions between housing condition, sucrose satiation group, or abstinence group, and the number of active-lever presses, inactive-lever presses, or sucrose deliveries on Training Days 7-10. Values were (Day 7-10 mean $\pm S E M$ ) active lever, $115.8 \pm 6.5$; sucrose deliveries, $59.6 \pm 2.8$; inactive lever, $4.5 \pm 0.5$ ). Locomotor activity did not differ between groups, but did significantly decrease $2.5 \%$ from Day 7 through Day 10 of training $[F(3,174)=4.7, p<.01$, 
$\left.\eta_{\mathrm{p}}{ }^{2}=.07\right]$; photobeam breaks averaged over Days 7-10, 1, $438.4 \pm 424.0$.

\section{Exposure phase}

Sucrose or water consumption measured in either the operant conditioning chamber or the home cage during the $17 \mathrm{~h}$ prior to testing is indicated in Fig. 2a. Overall, rats drank significantly more sucrose than water (four or five times more, depending on the condition) $[F(1,64)=251.5, p<.0001$, $\left.\eta_{\mathrm{p}}{ }^{2}=.8\right]$. We also observed a significant interaction of fluid type and forced-abstinence period $[F(1,62)=7.1, p<.01$, $\left.\eta_{\mathrm{p}}{ }^{2}=.1\right]$, but not of housing condition and forced-abstinence period. As is shown in Fig. 2b, rats that underwent 30 days of forced abstinence drank significantly more sucrose, but not water, than rats tested on the first day of forced abstinence (incubation of sucrose consumption).

\section{Testing phase}

Analysis of the compound-cue presentations revealed a significant interaction between forced-abstinence period and housing condition $\left[F(1,62)=11.8, p<.005, \eta_{\mathrm{p}}{ }^{2}=.16\right]$, and main effects of both forced-abstinence period, $F(1,64)=$ 20.3, $\left.p<.0001, \eta_{\mathrm{p}}{ }^{2}=.24\right]$ and housing condition, $F(1,64)=$ $\left.29.1, p<.0001, \eta_{\mathrm{p}}{ }^{2}=.31\right]$. Rats housed in the operant conditioning chamber for $17 \mathrm{~h}$ prior to the beginning of the testing session responded for significantly fewer cue presentations than did rats housed in their home cages prior to testing (Fig. 3a). This effect was significantly greater following 30 days of forced abstinence, indicated by a larger difference in cue presentations between housing groups at Day 30 than at
Day 1 (Fig. 3b). Similar to the incubation of sucrose consumption shown in Fig. 2, rats in the 30-day forced-abstinence condition received more cue presentations than did those tested on the first day of forced abstinence, regardless of housing condition (Fig. 3a). Access to sucrose did not affect compound-cue presentations $[F(1,64)=0.7, p=.39$, $\left.\eta_{\mathrm{p}}{ }^{2}=.01\right]$. Thus, our attempt to sate sucrose craving with sucrose was not effective at reducing responding to cue presentations, but extinction of the testing environment was (Fig. 3a).

Similar to that for cue presentations, the analysis of activelever pressing revealed a significant main effect of housing condition during the exposure period $[F(1,64)=16.2$, $\left.p<.0005, \eta_{\mathrm{p}}{ }^{2}=.2\right]$, and a significant main effect of days of forced abstinence $\left[F(1,64)=21.9, p<.0001, \eta_{\mathrm{p}}{ }^{2}=.26\right]$. However, unlike the statistically significant interaction between abstinence period and housing condition seen with cue presentations, the interaction for active-lever presses was only nearly statistically significant $[F(1,62)=3.4, p=.07$, $\left.\eta_{\mathrm{p}}{ }^{2}=.05\right]$. Extinction of the operant conditioning chamber reduced active-lever pressing, regardless of forced-abstinence period, yet active-lever pressing increased over days of forced abstinence (Fig. 4). Also similar to cue presentations, access to sucrose during the exposure period was without effect on active-lever pressing, $\left.F(1,64)=1.6, p=.21, \eta_{\mathrm{p}}{ }^{2}=.02\right]$.

Inactive-lever pressing was not found to differ between rats tested after one or 30 days of forced abstinence, or between the groups offered sucrose or water during the exposure period. We found a main effect of housing condition during the exposure period on inactive-lever pressing $[F(1,64)=20.6$, $\left.p<.0001, \eta_{\mathrm{p}}{ }^{2}=.24\right]$. However, no interaction was found between housing condition and days of forced abstinence $[F(1$,

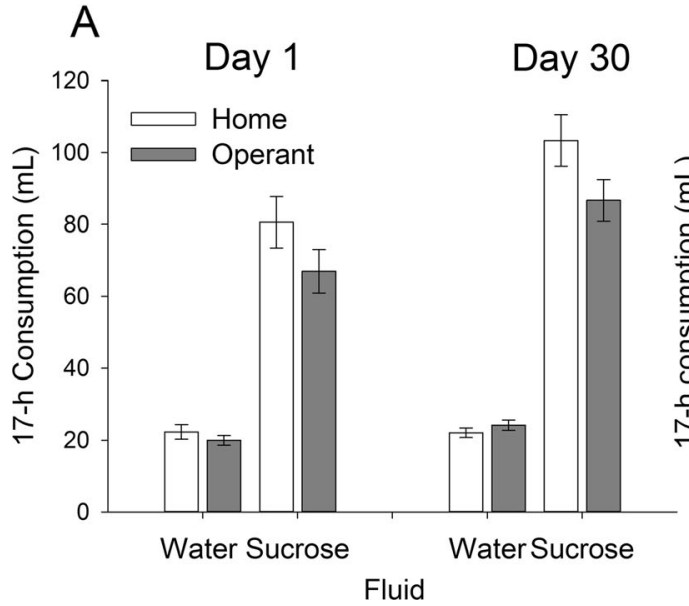

Fig. 2 Consumption of water or sucrose (satiation manipulation) during the 17-h exposure period. Forced-abstinence conditions are shown, indicated by "Day 1" or "Day 30." (a) All groups: Mean consumption ( \pm $S E M$ ) of either water or sucrose in the home cage (Home) or the operant conditioning chamber (Operant) at each time point in forced abstinence. (b) Data collapsed by housing conditions: Fluid type (water or sucrose) during satiation significantly interacted with the length of forced
B

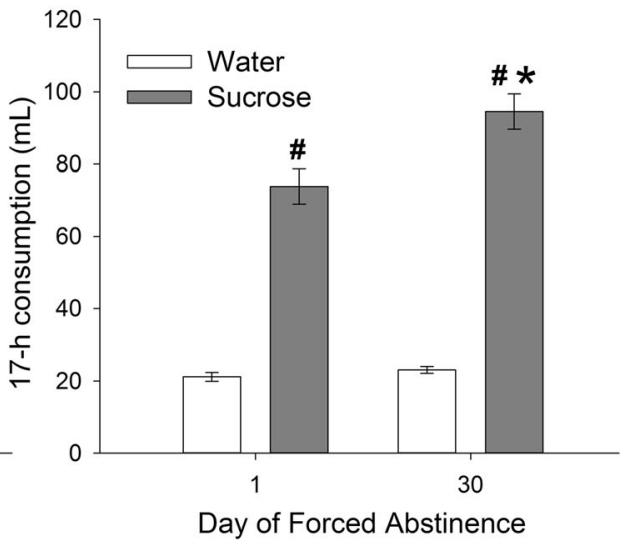

abstinence. Sucrose consumption was greater than water consumption at both time points, and sucrose consumption was greater on Day 30 than on Day 1. This increase indicates an "incubation" of sucrose consumption. ${ }^{\#}$ Overall significant effect of fluid type, $p<.05$. *Significant difference in sucrose consumption between Days 1 and 30 of forced abstinence, $p<.05$ 


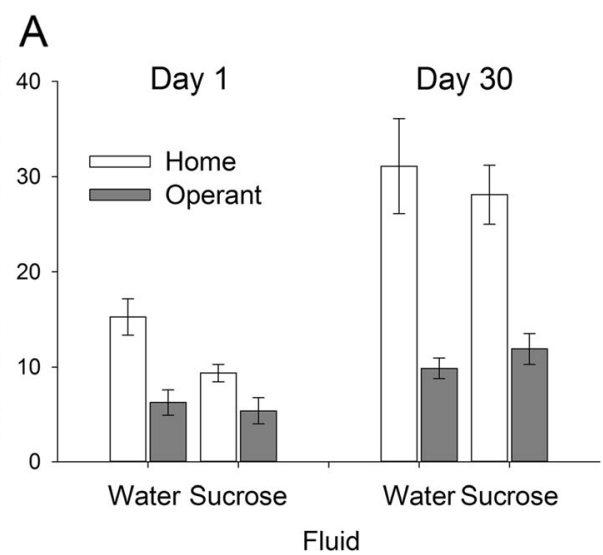

Fig. 3 Compound-cue presentations resulting from active-lever presses during testing. (a) All groups: Mean cue presentations ( \pm SEMs) of rats given access to either water or sucrose, in either the home cage (Home) or the operant conditioning chamber (Operant) during the 17-h exposure period. (b) Effect of housing on cue presentations. Data are collapsed by fluid types, showing a significant interaction of housing by day of forced abstinence. Rats housed in the operant conditioning chamber

62) $\left.=0.8, p=.37, \eta_{\mathrm{p}}{ }^{2}=.01\right]$ or between housing and sucrose satiation $\left[F(1,62)=0.3, p=.56, \eta_{\mathrm{p}}{ }^{2}=.01\right]$. Mean inactivelever responding was significantly reduced by extinction of the operant conditioning chamber on the first day of forced abstinence (home cage, $4.5 \pm 1.7$, and operant conditioning chamber, $1.2 \pm 0.4$ lever presses $/ 2 \mathrm{~h}$ ) and on the 30th day of forced abstinence (home cage, $6.8 \pm 1.2$, and operant conditioning chamber, $1.9 \pm 0.4$ lever presses $/ 2 \mathrm{~h}$; post-hoc $p$ s $<.05$ ). Locomotor activity was greater after 30 days of

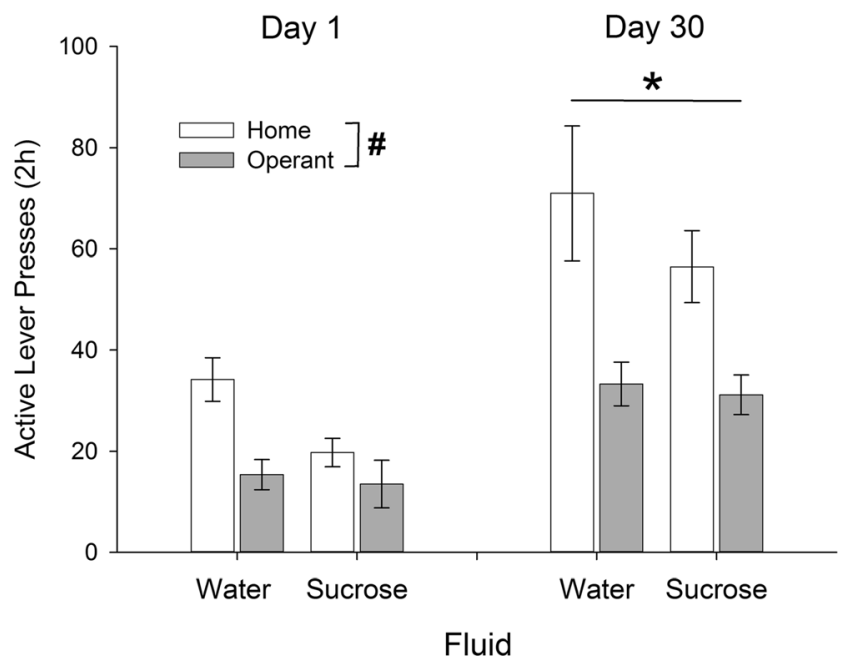

Fig. 4 Active-lever presses during testing: Mean active-lever presses ( \pm $S E M$ s) of rats given access to either water or sucrose in either the home cage (Home) or the operant conditioning chamber (Operant) during the 17-h exposure period. Rats that underwent 30 days of forced abstinence made significantly more active-lever presses than did rats tested immediately after training. Rats housed in the operant chamber during the exposure period were found to make significantly fewer active-lever presses than did rats housed in their home cage, regardless of the length of forced abstinence. * Main effect of forced-abstinence period, $p<.0001$. " Main effect of housing condition, $p<.0001$

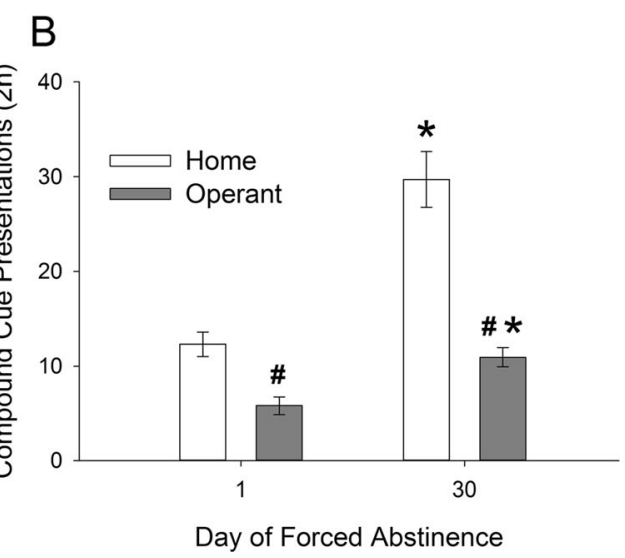

during the 17-h exposure period responded to significantly fewer compound-cue presentations than did rats housed in their home cages during this period. Rats tested after 30 days of forced abstinence responded to significantly more cue presentations than did rats tested after 1 day, indicating an incubation of responding. * Significant effects of length of forced abstinence, $p \mathrm{~s}<.05$. " Significant effects of housing condition on that day of forced abstinence, $p \mathrm{~s}<.05$

forced abstinence $\left[F(1,64)=4.4, p<.05, \eta_{\mathrm{p}}{ }^{2}=.06\right]$ and was reduced by extinction of the operant conditioning chambers $\left[F(1,64)=6.1, p<.05, \eta_{\mathrm{p}}{ }^{2}=.09\right]$. There were no significant interactions for locomotor behavior between abstinence period, housing condition, and/or fluid available during exposure. The photobeam breaks for the conditions according to the significant main effects were as follow: Day 1, 904.9 \pm 62.3 ; Day 30, 1,171.3 \pm 108.4 ; home cage, $1,197.6 \pm 69.2$; operant conditioning chamber, $886.6 \pm 104.6$.

\section{Discussion}

Context extinction attenuated lever pressing for a sucrosepaired cue in rats extinguished to the operant chamber, as compared to those extinguished to the home cage (Fig. 4). We also observed increases in both sucrose cue reactivity and sucrose consumption after a 30-day period of forced abstinence, similar to previous reports of incubated responding and consumption (Grimm et al., 2013; Harkness, Webb, \& Grimm, 2010). In contrast to context extinction, sucrose satiation did not reduce cue reactivity when rats were provided sucrose in either the home cages or operant conditioning chambers. Therefore, satiation of the primary reinforcer did not reduce responding for a cue previously associated with sucrose. These results suggest that contextual cues may have a greater influence on responding for a sucrose-paired cue than sucrose itself.

We previously demonstrated that satiety of sucrose consumption, by $17 \mathrm{~h}$ access to a sucrose solution immediately before operant testing, only slightly reduces extinction responding; there is no effect of sucrose satiation on responding for a sucrose-paired discrete cue after either one 
or 30 days of forced abstinence (Grimm, Fyall, \& Osincup, 2005). In our previous report, we speculated that satiety to sucrose does not greatly affect the conditioned motivation that underlies relapse. The present findings support this hypothesis. Rats consumed three to four times more sucrose solution by volume than water controls during exposure (Fig. 2), yet their sucrose cue reactivity was similar to that of controls (Figs. 3 and 4).

Extinction of contextual cues, on the other hand, reduced lever responding for the cue previously associated with sucrose. One explanation for this effect is that the excitatory power of the chamber, due to its past association with sucrose self-administration availability, was reduced by prolonged exposure of the subject to it without the availability of selfadministration cues (response-contingent sucrose and associated cues including the lever and tone+light cue). Therefore, in the test session, rats only showed behavior elicited by the sucrose self-administration cues. Another explanation is similar, but emphasizes the connections between stimuli in the self-administration context. Bindra (1972) suggested that the entirety of the conditioning context functions as a "complete CS [conditioned stimulus]," and therefore omission (or extinction) of an element would reduce the overall power of the complete CS to elicit responding. That is, lever pressing following context extinction may represent the remaining portion of responding engendered by the tone + light discrete cue alone. Marlin and Miller (1981) and Marlin (1982) proposed a similar idea, but emphasized the role of context as an especially important CS. In addition, they provided evidence supporting the idea that the motivational valence of CS elements are linked. For example, they described how extended exposure (habituation) to a shock-predictive context (argued to be a CS itself) led to decreased fear elicited by the shockassociated CS. It is interesting to note, however, that inasmuch as satiety should have decreased motivation to seek sucrose, the satiety manipulation was only marginally effect (see the next paragraph) and only on Day 1 of forced abstinence. Therefore, if CS elements are tied to motivational state, the present results indicate that the ability to observe the impact of the associations depends on length of abstinence. Discerning which of the above explanations best fits the present data is not possible without further experimentation.

Incubation of craving (Grimm, Hope, Wise, \& Shaham, 2001) is described as an abstinence-dependent increase in responding for a cue associated with food or drug (Grimm et al., 2011) and, recently, has been reported with the nonfood or drug reinforcer saccharin (Aoyama, Barnes, \& Grimm, 2014). Incubation of craving has been observed in human addicts (Bedi et al., 2011; Li et al., 2014; Nava et al., 2006; Wang et al., 2014) and may be a key factor in high rates of relapse to drugs and food in humans. In the present study, sucrose cue reactivity was also significantly greater after one month of forced abstinence. Only context extinction significantly interacted with incubation, reducing lever pressing by operant conditioning chamber-housed animals to a greater extent on Day 30 than on Day 1 of forced abstinence (Fig. 3b). Figures 3a and 4 illustrate apparently reduced responding on Day 1 for sucrose-satiated subjects, yet the interaction between day and fluid was not statistically significant (see the Results section). To test for an effect of sucrose satiation, exploratory Bonferroni-corrected $t$ tests were performed for cue presentations and active-lever responses comparing water versus sucrose. We found that the sucrose satiation manipulation reduced responding and cue presentations on Day $1(p<.01)$. With increased statistical power we might have detected this effect in the ANOVA. Regardless, the $t$ test results confirm our previous finding that sucrose satiation decreased initial extinction responding (essentially, the cue reactivity testing in the present study), but not responding for a discrete cue (Grimm et al., 2005). As we noted above, neither Grimm et al. (2005) or the present study showed that sucrose satiation reduced sucrose cue reactivity after one month of forced abstinence.

In a previous study, we observed a time-dependent increase in sucrose consumption between Days 1 and 7 of forced abstinence, but not between Days 1 and 30 (Grimm et al., 2005). However, we have since consistently observed incubation of sucrose consumption over 30 days, either in the home cages (present study) or in the operant conditioning chamber (present study; Grimm et al., 2013). Procedural differences may account for the discrepancy between Grimm et al. (2005) and these two more recent studies. For example, rats selfadministered sucrose for $6 \mathrm{~h}$ /day in Grimm et al. (2005), versus $2 \mathrm{~h} /$ day in the present study and Grimm et al. (2013). This inconsistency between studies indicates that incubation of sucrose consumption is not necessary for incubation of sucrose cue reactivity. In addition, self-administration cues are not necessary for incubation of sucrose consumption, because incubation of consumption occurred in the home cage (see the Fig. 2a home cage extinction sucrose groups and Fig. 2b).

Future studies could examine the neurobiological substrates of the extinction effect that we observed in particular brain regions that might have mediated the observed dissociation between primary and secondary reinforcement. Targets for future investigations might include the nucleus accumbens (primary reinforcement; Wise, 2004), hippocampus (conditioned context; Marchant, Kaganovsky, Shaham, \& Bossert, 2014), and the basolateral amygdala (conditioned discrete cue; Weiss et al., 2000). Finally, although a $10 \%$ sucrose solution is one of the most widely used concentrations for rodent selfadministration studies, and was used by both Grimm et al. (2005) and the present study, others have used solutions as low as $4 \%$ (Galarce, Crombag, \& Holland, 2007) or as high as $20 \%-75 \%$ (Samson \& Chappell, 1999), either of which extreme could have resulted in different levels of cue reactivity following the exposure manipulations used in the present 
study. Examining the potential role of magnitude of reinforcement in cue reactivity could be a manipulated variable in future studies.

\section{Conclusion}

The present results indicate that context extinction reduces cue reactivity. These results support the conclusion that the selfadministration context, including discrete cues paired with self-administration, maintains a powerful influence on conditioned responding even after extended forced abstinence. In contrast, the primary reinforcer itself has little control over conditioned responding, especially after extended forced abstinence. Craving and relapse can be triggered by exposure to stimuli previously associated with drugs or food (Carter \& Tiffany, 1999; Childress et al., 1999; Epstein et al., 2009; Jansen et al., 2003; Sobik, Hutchison, \& Craighead, 2005). On the basis of our findings reported here, the extinction of drug- or food-associated contexts may be an effective tool for reducing craving and relapse in human addicts.

Author note J.W.H. is now at the Department of Behavioral Neuroscience, Oregon Health and Science University. S.W. is now at the Department of Psychological and Brain Sciences, University of California, Santa Barbara. The authors have no personal or professional associations that could be considered conflicts of interest. This research was supported by NIH Grant Number R15 DA016285-03 and by Western Washington University. The authors thank Amber Fyall for helping with data collection.

\section{References}

Aoyama, K., Barnes, J., \& Grimm, J. W. (2014). Incubation of saccharin craving and within-session changes in responding for a cue previously associated with saccharin. Appetite, 72, 114-122. doi:10. 1016/j.appet.2013.10.003

Bedi, G., Preston, K. L., Epstein, D. H., Heishman, S. J., Marrone, G. F., Shaham, Y., \& de Wit, H. (2011). Incubation of cue-induced cigarette craving during abstinence in human smokers. Biological Psychiatry, 69, 708-711. doi:10.1016/j.biopsych.2010.07.014

Bindra, D. (1972). A unified account of classical conditioning and operant training. In A. H. Black \& W. F. Prokasy (Eds.), Classical conditioning II: Current research and theory (pp. 453-481). New York: Appleton-Century-Crofts.

Bouton, M. E., \& Bolles, R. C. (1979). Role of conditioned contextual stimuli in reinstatement of extinguished fear. Journal of Experimental Psychology: Animal Behavior Processes, 5, 368378. doi:10.1037/0097-7403.5.4.368

Carter, B. L., \& Tiffany, S. T. (1999). Meta-analysis of cue-reactivity in addiction research. Addiction, 94, 327-340.

Childress, A. R., Mozley, P. D., McElgin, W., Fitzgerald, J., Reivich, M., \& O'Brien, C. P. (1999). Limbic activation during cue-induced cocaine craving. American Journal of Psychiatry, 156, 11-18.

Crombag, H. S., Grimm, J. W., \& Shaham, Y. (2002). Effect of dopamine receptor antagonists on renewal of cocaine seeking by reexposure to drug-associated contextual cues. Neuropsychopharmacology, 27, 1006-1015. doi:10.1016/S0893-133X(02)00356-1
Crombag, H. S., \& Shaham, Y. (2002). Renewal of drug seeking by contextual cues after prolonged extinction in rats. Behavioral Neuroscience, 116, 169-173.

Dagher, A. (2009). The neurobiology of appetite: hunger as addiction. International Journal of Obesity, 33(Suppl. 2), S30-S33. doi:10. 1038/ijo.2009.69

Epstein, D. H., Willner-Reid, J., Vahabzadeh, M., Mezghanni, M., Lin, J. L., \& Preston, K. L. (2009). Real-time electronic diary reports of cue exposure and mood in the hours before cocaine and heroin craving and use. Archives of General Psychiatry, 66, 88-94. doi:10.1001/ archgenpsychiatry.2008.509

Freeman, T. P., Morgan, C. J., Beesley, T., \& Curran, H. V. (2012). Drug cue induced overshadowing: selective disruption of natural reward processing by cigarette cues amongst abstinent but not satiated smokers. Psychological Medicine, 42, 161-171. doi:10.1017/ S0033291711001139

Galarce, E. M., Crombag, H. S., \& Holland, P. C. (2007). Reinforcerspecificity of appetitive and consummatory behavior of rats after Pavlovian conditioning with food reinforcers. Physiology and Behavior, 91, 95-105. doi:10.1016/j.physbeh.2007.01.021

Grimm, J. W., Fyall, A. M., \& Osincup, D. P. (2005). Incubation of sucrose craving: effects of reduced training and sucrose pre-loading. Physiology and Behavior, 84, 73-79. doi:10.1016/j.physbeh.2004. 10.011

Grimm, J. W., Harkness, J. H., Ratliff, C., Barnes, J., North, K., \& Collins, S. (2011). Effects of systemic or nucleus accumbensdirected dopamine D1 receptor antagonism on sucrose seeking in rats. Psychopharmacology, 216, 219-233. doi:10.1007/s00213011-2210-y

Grimm, J. W., Hope, B. T., Wise, R. A., \& Shaham, Y. (2001). Neuroadaptation. Incubation of cocaine craving after withdrawal. Nature, 412, 141-142. doi:10.1038/35084134

Grimm, J. W., Weber, R., Barnes, J., Koerber, J., Dorsey, K., \& Glueck, E. (2013). Brief exposure to novel or enriched environments reduces sucrose cue-reactivity and consumption in rats after 1 or 30 days of forced abstinence from self-administration. PLOS ONE, 8, e54164. doi:10.1371/journal.pone.0054164

Harkness, J. H., Webb, S., \& Grimm, J. W. (2010). Abstinence-dependent transfer of lithium chloride-induced sucrose aversion to a sucrosepaired cue in rats. Psychopharmacology, 208, 521-530. doi:10. 1007/s00213-009-1755-5

Jansen, A., Theunissen, N., Slechten, K., Nederkoorn, C., Boon, B., Mulkens, S., \& Roefs, A. (2003). Overweight children overeat after exposure to food cues. Eating Behaviors, 4, 197-209. doi:10.1016/ S1471-0153(03)00011-4

Li, P., Wu, P., Xin, X., Fan, Y. L., Wang, G. B., Wang, F., . . Lu, L. (2014). Incubation of alcohol craving during abstinence in patients with alcohol dependence. Addiction Biology, 20, 513-522. doi:10. 1111/adb. 12140

Marchant, N. J., Kaganovsky, K., Shaham, Y., \& Bossert, J. M. (2014). Role of corticostriatal circuits in context-induced reinstatement of drug seeking. Brain Research. doi:10.1016/j.brainres.2014.09.004

Marlin, N. A. (1982). Within-compound associations between the context and the conditioned stimulus. Learning and Motivation, 13, 526531.

Marlin, N. A., \& Miller, R. R. (1981). Associations to contextual stimuli as a determinant of long-term habituation. Journal of Experimental Psychology: Animal Behavior Processes, 7, 313-333. doi:10.1037/ 0097-7403.7.4.313

Nava, F., Caldiroli, E., Premi, S., Lucchini, A. (2006). Relationship between plasma cortisol levels, withdrawal symptoms and craving in abstinent and treated heroin addicts. Journal of Addictive Diseases, 25(2), 9-16.

PHS (2002) Policy on humane care and use of laboratory animals. http:// grants.nih.gov/grants/olaw/references/phspol.htm. Accessed 6 July 2015. 
Preston, K. L., Vahabzadeh, M., Schmittner, J., Lin, J. L., Gorelick, D. A., \& Epstein, D. H. (2009). Cocaine craving and use during daily life. Psychopharmacology, 207, 291-301. doi:10.1007/s00213-0091655-8

Rescorla, R. A. (2003). Contemporary study of Pavlovian conditioning. Spanish Journal of Psychology, 6, 185-195.

Robbins, T. W., Ersche, K. D., \& Everitt, B. J. (2008). Drug addiction and the memory systems of the brain. Annals of the New York Academy of Sciences, 1141, 1-21. doi:10.1196/annals.1441.020

Samson, H. H., \& Chappell, A. M. (1999). Effects of microinjection of the D2 dopamine antagonist raclopride into the ventral tegmental area on ethanol and sucrose self-administration. Alcoholism: Clinical and Experimental Research, 23, 421-426. doi:10.1111/j. 1530-0277.1999.tb04132.x

Shalev, U., Grimm, J. W., \& Shaham, Y. (2002). Neurobiology of relapse to heroin and cocaine seeking: A review. Pharmacological Review, $54,1-42$.

Sobik, L., Hutchison, K., \& Craighead, L. (2005). Cue-elicited craving for food: A fresh approach to the study of binge eating. Appetite, 44, 253-261. doi:10.1016/j.appet.2004.12.001

Todd, T. P., Winterbauer, N. E., \& Bouton, M. E. (2012a). Contextual control of appetite. Renewal of inhibited food-seeking behavior in sated rats after extinction. Appetite, 58, 484-489. doi:10.1016/j. appet.2011.12.006

Todd, T. P., Winterbauer, N. E., \& Bouton, M. E. (2012b). Effects of the amount of acquisition and contextual generalization on the renewal of instrumental behavior after extinction. Learning \& Behavior, 40, 145-157. doi:10.3758/s13420-011-0051-5

Volkow, N. D., \& Wise, R. A. (2005). How can drug addiction help us understand obesity? Nature Neuroscience, 8, 555-560. doi:10.1038/ nn1452

Wang, Y., Wang, H., Li, W., Zhu, J., Gold, M. S., Zhang, D., .. W Wang, W. (2014). Reduced responses to heroin-cue-induced craving in the dorsal striatum: Effects of long-term methadone maintenance treatment. Neuroscience Letters, 581, 120-124. doi:10.1016/j.neulet. 2014.08.026

Weiss, F., Maldonado-Vlaar, C. S., Parsons, L. H., Kerr, T. M., Smith, D. L., \& Ben-Shahar, O. (2000). Control of cocaine-seeking behavior by drug-associated stimuli in rats: Effects on recovery of extinguished operant-responding and extracellular dopamine levels in amygdala and nucleus accumbens. Proceedings of the National Academy of Sciences, 97, 4321-4326.

Wise, R. A. (2004). Dopamine, learning and motivation. Nature Reviews Neuroscience, 5, 483-494. 